\title{
Selection of Mulberry Genotypes for Rainfed Conditions through Principal Component Analysis
}

\author{
K. Suresh*, H. Yallappa, Anil Pappachan, Maloy Laskar, \\ G. R. Manjunath, D. Chakravarthy and V. Sivaprasad \\ Central Sericultural Research and Training Institute, Berhampore, West Bengal, India \\ *Corresponding author
}

\section{A B S T R A C T}

\section{Keywords}

Mulberry, Leaf yield, Rainfed,

Principal

component and

AMMI stability

\section{Article Info}

Accepted:

18 December 2020

Available Online:

10 January 2021
Development of high yielding varieties with acceptable level of stability is an important objective of mulberry breeding programme. Thirty selected genotypes and three check varieties were evaluated for leaf yield and its components along with physio-biochemical parameters under rainfed condition. Principal component analysis generated twenty eight PCs, out of which nine PCs exhibited more than one Eigen value explaining $78.6 \%$ of variability among the traits studied. Based on biplot analysis, leaf area, leaf moisture, moisture retention capacity, length of longest shoot, primary shoots per plant, total shoots length, total soluble sugars, leaf area index and chlorophyll $\mathrm{a} / \mathrm{b}$ ratio were identified as important yield contributing traits under rainfed condition. The AMMI analysis of variance across three seasons revealed that the leaf yield was significantly $(\mathrm{p}<0.01)$ affected by seasons/environments $(\mathrm{E})$, genotypes $(\mathrm{G})$ but not by $\mathrm{G} \times \mathrm{E}$ interaction. Environment significantly explained about $93.2 \%$ of the total sum of squares indicating that diverse seasonal environments caused most of the variations in yield. AMMI stability value (ASV) identified genotype PYD 21, PYD 06, PYD 04, PYD 28, and PYD 01 as the most stable, while PYD 13, PYD 20 as least stable. From the present investigation, Genotypes PYD 08, PYD 15, PYD 27, PYD 01 and PYD 07 were identified as high yielding with better stability across seasons over C-2038 and could be further evaluated to identify suitable variety for rainfed sericulture.

\section{Introduction}

Mulberry leaf is the major economical component in Sericulture. Its quality and quantity has a direct bearing on cocoon harvest. India ranks second after China in mulberry silk production with around 25,345 MT from an area of 2.35 lakh hectares (www.csb.org.in/2018-19). The tremendous growth of population in India has increased the demand for silk and requires a production of 50,000 MT by the year 2025. Improved mulberry and silkworm variety/breed play a pivotal role in further enhancing productivity of Sericulture.

Mulberry leaf productivity is one of the principal factors that decide the sustainability and profitability of sericulture (Ashiru, 2002 and Doss et al., 2012). Although, a good 
number of high yielding mulberry varieties have been developed but still the productivity of mulberry leaf-cocoon-silk remains low. Development of high yielding mulberry varieties through various breeding approaches is a continuous process. Mulberry is predominantly cultivated as rainfed crop and low leaf productivity with poor quality is the major constraint. Further climate change causing drought stress in most of the rainfed areas has considerable effects on silk production. The present scenario of Sericulture enterprise needs demands new varieties appropriate for different agroclimatic situations. Hence, development of high yielding mulberry genotypes suitable for rainfed area remains a top priority.

Improvement of mulberry yield both in quantitative and qualitative aspect is the long term goal of mulberry breeders. Mulberry leaf yield is a complex trait and is contributed by a number of component traits that are highly influenced by environment and management practices. The selection of superior genotypes of mulberry is an intricate process, thus exploratory multivariate techniques can be applied to select genotypes by analyzing the morpho-physio-biochemical traits altogether increasing the success of the genetic improvement programme. Principal component analysis (PCA) is an exploratory tool that transforms a number of correlated variables into a smaller number of uncorrelated variables called principal components (Muniraja et al., 2011). PCA results in generation of a $2 \mathrm{D}$ scatter plot of genotypes and component traits, whose geometrical distances helps in identification of correlated traits and genetically similar genotypes (Mohammadi, 2003). PCA is a well-known method of dimension reduction that reduce a large set of variables into a small set, that still contains most of the information in the large set (Massay,1965 and Jolliffie,1986). PCA has many applications and among which computing an index by assigning the weights is one. PCA-based selection index targets the simultaneous improvement of several traits at the same time, including the leaf yield.

The variation in yield and its components due to changing environment is commonly referred to as genotype $\times$ environment interaction (GEI). It results from the differential expression of genotypes over the environments which complicate the process of selecting superior genotypes. Effects of genotype, environment and genotype $\times$ environment interaction determine the phenotypic performance, its general and specific adaptation to different environments (Falconer and Mackey, 1996). Identifying a phenotypically stable variety is very important from the point of increasing mulberry production. This information is required for planning better selection strategies and to identify genotypes with better stability across environments (Gauch and Zobel, 1996 and Kang, 1998).Additive main effects and multiplicative interaction (AMMI) analysis is widely used for GEI investigation and it clearly separates main effects and interaction effects, which helps in understanding stability of genotypes to support the breeding programme (Crossa et al., 1990; Gauch and Zobel, 1997). It combines ANOVA for the genotype and environment main effects with principal components analysis of GEI (Gauch and Zobel, 1996). However AMMI model does not make provision for a quantitative stability measure. AMMI stability value (ASV) proposed by Purchase (1997) quantifies and rank genotypes according to their yield stability. ASV is the distance from zero in a two dimensional scatter of IPCA 1 scores against IPCA 2 scores. The aim of this investigation was to identify superior genotypes with better yield stability across seasons for rainfed condition. 


\section{Materials and Methods}

The experiment was conducted at the experimental farm of Central Sericultural research and training institute, Berhampore West Bengal (Latitude: 24'5'28.01"N and Longitude: $\left.88^{\circ} 15^{\prime} 56.37 " \mathrm{E}\right)$ during 2017 to 2019. Thirty test genotypes derived from different parental crosses (Baragarh-2 $\times$ Thailand: PYD 1 to 3; Baragarh- $2 \times$ UP -23: PYD 4 to 9; Khakad $2 \times$ V1: PYD 10 \& 11; Hosur C8 $\times$ Thailand: PYD 12 \& 13; Jalalgarah $3 \times$ S13: PYD 14 to 17 ; Chirayinkizh $\times$ S1: PYD 18 \& 19; Saranth $2 \times$ S13: PYD 21 to 25 ; ERRC $32 \times$ Lazuraso: PYD 26 and Baragarh $2 \times$ Madhopur 4: PYD 27 to 30 ) and three check varieties viz., C2038, S-1635 and C-1730 were evaluated for their suitability under rainfed conditions. The study was carried out for three seasons across two years under randomized block design (RBD) with two replications. Six month old saplings were transplanted in a plot size of 1.5 $\mathrm{m} \times 1.8 \mathrm{~m}$ with $90 \mathrm{~cm} \times 90 \mathrm{~cm}$ spacing. One border row of S-1635 was maintained to minimize the border effects. Plants were trained for low bush system of one foot crown height and allowed to establish for one year. From second year, plants were pruned as per commercial crop schedules of the region. Standard agronomic practices and plant protection measures were adopted to raise a healthy crop (Ray et al., 1973).

Observations were recorded on average of five plant basis for twelve yield and yield contributing traits as per standard procedures. Further, data was recorded on average of three plant basis for twelve physiological and biochemical parameters such as total chlorophyll content (Arnon et al., 1949), chlorophyll $\mathrm{a} / \mathrm{b}$ ratio, total soluble protein (Lowry et al., 1951), total soluble sugars (Morris et al., 1948), Leaf proline content (Bates et al., 1973), Epicuticular wax (Ebercon et al., 1977), Relative water content
(Barrs and Weatherly, 1962), water loss rate of excised leaves $\left(\mathrm{mg} \mathrm{g}^{-1} \mathrm{~h}^{-1}\right)$, stomata frequency $\left(\mathrm{No}^{\prime} \mathrm{s} \mathrm{mm}^{-2}\right)$, leaf thickness $(\mu \mathrm{m})$, chlorophyll content index by hand held Chlorophyll meter(Opti-Sciences, CCM 200) and leaf area index.

The mean data obtained was subjected to pooled analysis of variance, considering both seasons and genotypes as fixed using OPSTAT tool (Sheoran, HAU, Hisar). The principal component analysis method explained by Harman (1976) was followed in the extraction of the components. PCA and biplot graphical display was performed using $\mathrm{R}$-studio software. PCA based selection index was constructed using computed principal components where in data was normalized and weights were assigned based on the PCs having Eigen value greater than one. The sum products of absolute value of factor loading values with their respective Eigen values give the weights for each variable. PCA based index for each genotype is computed by dividing the sum product of normalized observed value with their respective assigned weights and then divided by sum of the all weights (Mahida and Sendhil, 2017). Based on the index values, the ranks were assigned to each genotype for selection of superior genotypes. The AMMI model developed by Gabriel (1971) has been applied to understand $\mathrm{G} \times \mathrm{E}$ interaction and used for identifying stable genotypes and environment. AMMI analysis of $\mathrm{G} \times \mathrm{E}$ interaction was performed using R-studio software (Crossa et al., 1990).

\section{Results and Discussion}

\section{ANOVA and mean performance of test genotypes under rainfed condition}

The mean sum of squares for leaf yield and its components among the genotypes for three seasons over two years are presented in Table 1. Significant differences were 
observed between genotypes for most of the traits studied indicating the presence of sufficient amount of variability and provided scope for selection. Most of the studied traits, except for specific leaf area, leaf moisture content, moisture retention capacity and leaves per meter shoot showed statistically significant interaction indicating the impact of environment in the expression of these traits. Three way interactions for genotypes $x$ years $x$ seasons revealed the influence of all these factors in expression of the most of traits studied. The study revealed that genotypes responded differently in different seasons and possibility of selecting genotypes with high or low stability.

The descriptive statistics (minimum, maximum, standardized range and coefficient of variation) were computed from pooled data of three seasons over two years for 12 quantitative traits (Table 1). Based on standardized range, leaf yield, fresh leaf weight, leaf area and leaf fall at harvest were found to be the most variable traits. The lowest variations were observed for leaf moisture and moisture retention capacity and all the other traits showed intermediate values. C-2038 performed better than the other two check varieties in most of the parameters studied and the performance of test genotypes was compared with C-2038 for their suitability to rainfed conditions. Mean weight (1.756 to $4.236 \mathrm{~g})$ and area (97.14 to $215.40 \mathrm{~cm}^{2}$ ) of fresh leaves collected from three positions of longest shoot varied significantly. All the test genotypes produced medium sized leaves which were smaller than the variety C-2038. Plants with low Specific Leaf Area (SLA) have thicker leaves with higher chlorophyll per unit leaf area and greater photosynthetic capacity compared with thinner leaves (Richardson et al., 2002). SLA varied from 176.5 to $224.25 \mathrm{~cm}^{2} \mathrm{~g}^{-1}$ wherein seven genotypes recorded significantly lower values than C-
2038.Twenty four to thirty leaves were present per meter length of shoot and 18 genotypes recorded longer shoots compared to check C-2038. Primary shoots and total shoots length per plant varied from 7 - 10 No's and $719-1155 \mathrm{~cm}$, respectively. Leaf to shoot weight ratio ranged from 50.55 to 57.69 $\%$ and eleven genotypes recorded significantly higher values than C2038. All the test genotypes were found to have less leaf fall during harvest compared the variety C2038. Leaf yield per plant varied from 1.09 $-1.96 \mathrm{~kg} / \mathrm{yr}$ and ten genotypes were found to be significantly superior to check C2038 (1.60kg/yr). Genotype PYD 08, PYD 27, PYD 01, PYD 21 and PYD 04 exhibited more than $10 \%$ higher leaf yield over C-2038.

Nutritional content of mulberry leaves greatly influence the growth and development of silkworm larvae, which in turn affects the quality and quantity of cocoon/silk produced (Fonseca, et al., 1990; Purohit and Pavankumar, 1996). Highest total soluble protein content was observed in PYD 10 (43.65 $\mathrm{mg} \mathrm{g}^{-1}$ ) and eight genotypes showed significantly higher protein content over C2038 (35.25 $\mathrm{mg} \mathrm{g}^{-1}$ ). Higher soluble sugar content was noticed in the leaves of PYD 18 while wide variation (31.49-49.88 $\mathrm{mg} \mathrm{g}^{-1}$ ) was observed among test genotypes. Horie and Watanabe (1980) reported that total soluble sugar and total soluble protein are the most important biochemical constituents of mulberry leaf that positively favors the silkworm larval growth and cocoon yield. Chlorophyll content is crucial for quantifying the photosynthetic efficiency rate in mulberry (Sujathamma and Dandin, 2000) and is an essential constituent in assessing quality of foliage. Total chlorophyll content in leaf varied from 1.81 to $2.81 \mathrm{mg} \mathrm{g}^{-1}$ among the genotypes studied. The highest total chlorophyll content was observed in C-2038 which is associated with enhanced photosynthetic efficiency that leads to better 
yield and quality of the leaf. Leaf moisture content is positively correlated with palatability and assimilability of nutrients by silkworm (Parpiev, 1968). Most of the genotype was found to have desirable leaf moisture (72.64-76.59 \%) and moisture retention capacity after 6 hours (69.32-76.35 $\%$ ). Lamina thickness of fully expanded leaf ranged from $124-162 \mu \mathrm{m}$ and PYD 01, PYD 10, PYD 12, PYD 15, PYD 17, PYD 18, PYD 27 and PYD 29 found to have thicker lamina than C-2038. Several studies have revealed the importance of biochemical quality parameters for selection of superior genotypes in mulberry (Fotadar et al., 1989; Chaluvachari and Bongale, 1995; Thirumalaisamy et al., 2009 and Ramamoorthy et al., 2018).

Physiological traits are the common indicators to estimate crop biomass production potential (Cao et al., 2019). Significant differences were observed among the tested genotypes for leaf proline, epicuticular wax, relative water content, water loss rate, chlorophyll a/b ratio, stomata frequency, chlorophyll content index and leaf area index. Leaf proline (283-2344 $\left.\mu \mathrm{g} \mathrm{g}^{-1}\right)$ and epicuticular wax (312-346 $\mu \mathrm{g} \mathrm{cm}^{-}$ $\left.{ }^{2}\right)$ are the important parameters of drought tolerance and exhibited wide variations. Twelve genotypes recorded significantly higher proline and epicuticular wax content compared to C2038. Relative water content (RWC) is the most appropriate measure of plant water status. PYD 23 (91.95\%) and PYD 10 (65.53\%) recorded highest and lowest values, respectively. Water loss rate of excised leaves among test genotypes varied three fold (209-610 $\mathrm{mg} \mathrm{g}^{-1}$ $\mathrm{h}^{-1}$ ) and twenty genotypes recorded significantly lower water loss rate compared to C-2038. Adjustment of the Chlorophyll a/b ratio was apparently an integral feature of acclimation to high light conditions and low $\mathrm{N}$ availability (Kitajima and Hogan, 2003). Nineteen genotypes were observed to have significantly higher Chlorophyll a/b ratio among the test genotypes studied (1.37-3.73). Two fold variations was observed for stomata frequency (648-1434 No. $\mathrm{mm}^{-2}$ ) and seventeen genotypes recorded lower number of stomata compared to C2038. Leaf area index on 25 days after sprouting recorded three fold variation and all genotypes were found to have less LAI than C-2038. The Coefficient of variation (CV) ranged from 4.17- $32.68 \%$ indicating the wide range of variation which might be due to divergent genotypes included in the study. The presence greater variability among the genotypes provides scope for making further improvement through selection. Similar results on presence of high variability was reported by Tikader and Rao, 2001; Tikader et al., 2004; Doss et al., 2006; Banerjee et al., 2007; Mallikarjunappa et al., 2008; Vijayashekara, 2009; Biradar et al., 2015; Suresh et al., 2017; Chanotra et al., 2019 and Rahman and Islam, 2020.

\section{Principal component analysis and PCA based selection index for identifying superior genotypes}

To determine the patterns of variation and to detect the structure in the relationships between traits, PCA was carried out considering all of the 28 quantitative traits simultaneously (Table 2). Out of twenty eight PCs formed, only 9 PCs exhibited more than one Eigen value and showed about $78.6 \%$ variability. Eigen values above one generate components with significant quantities of information of the original variables.

The PC1 accounted for maximum proportion of total variability in the set of all variables studied and remaining components accounted for progressively lesser and lesser variation. PC1 was responsible for about $22 \%$ of the variation and was mainly contributed by fresh 
leaf weight (0.378), leaf area (0.374), leaf fall at harvest (0.293), leaf area index (0.309) and water loss rate $(0.287)$. Whereas second PC $(13.8 \%)$ was more related to yield attributing traits such as specific leaf area (0.364), leaf moisture (0.354), leaves per meter shoot (0.133), total shoot length (0.327) and leaf yield per plant (0.322).

The first two principal components (PC1 and PC2) were characterized by the variables such as fresh leaf weight, leaf area, specific leaf area, leaf moisture content, leaves per meter shoot, total shoots length, leaf area index and leaf yield per plant. It was possible to discriminate individual genotypes PYD 01, PYD 02, PYD 03, PYD 04, PYD 08, PYD 09, PYD 11, PYD 21, PYD 23, PYD 26 and PYD 28 for such traits (Table 3). The third principal component (PC3) explained 10.1\% of variation and was associated mainly with moisture retention capacity (0.293), total soluble sugars (0.293) and leaf proline content (0.295). PC4 explained $7 \%$ of total variation and primary shoots per plant (0.293), Bacterial leaf spot (0.362), tukra infestation (0.161) were the major contributing traits. PC5 mainly consist of total soluble protein (0.592), epicuticular wax content (0.407), chlorophyll $\mathrm{a} / \mathrm{b}$ ratio $(0.315)$ and explained $6.5 \%$ of variation.

The sixth component (PC6) was more related to relative water content (0.435), Pseudocercospora leaf spot (0.069), Myrothecium leaf spot (0.196) whereas PC7 was closely related to length of the longest shoot (0.458) and chlorophyll content index (0.329). Lastly in PC8, leaf to shoot ratio (0.429), total chlorophyll content (0.295), lamina thickness (0.488) and in PC 9 stomata frequency $(0.601)$ were dominated.

Biplot analysis of thirty genotypes and three checks formed by the components PC1 and PC2 retained a total $35.8 \%$ of the original variance. Length of arrows showed the proportion of contribution in the principal component and direction of arrows indicated whether the proportion was positive or negative as shown in Fig. 1.

From these results it can be concluded that characters like leaf weight, leaf area, leaf moisture, moisture retention capacity, length of longest shoot, primary shoots per plant, total shoots length, total chlorophyll, total soluble sugars, epicuticular wax, lamina thickness, leaf area index and chlorophyll a/b ratio had direct effect on mulberry yield under rainfed condition. Banerjee et al., (2011) identified six PCs which explained $>88 \%$ of total variation among twenty five mulberry accessions for 22 traits studied and traits such as longest shoot length, leaf area, inter-nodal distance, green and dry leaf weight, root volume, fresh and dry root weight were reported to have direct effect on cocoon yield. In this process, the genotypes PYD 01, PYD 04, PYD 08 and PYD 27 which were farther from the axis origin, outward the circumference and in the direction of the vectors (arrows) corresponding to the leaf yield were identified as superior. Similar results were also reported by Chanotra et al., (2019)

The top principal component scores (PC scores) for all the traits were estimated in 33 genotypes and presented in table 3. High PC score for a specific genotype in a particular component denotes high values for the variables in that particular genotype. PYD 08 had the highest score 5.321 value followed by C-2038 (4.170), PYD 26 (3.610), PYD 09(3.545), S-1635(3.292), PYD 23(2.705) and PYD 11(1.577), indicating that they had high value for fresh leaf weight, leaf area, moisture retention of leaves, leaves per meter shoot, leaf fall at harvest, leaf area index, chlorophyll $\mathrm{a} / \mathrm{b}$ ratio and water loss rate. 
Table.1 Pooled analysis of variance over 3 seasons and descriptive statistics for leaf yield and its component traits

\begin{tabular}{|c|c|c|c|c|c|c|c|c|c|c|c|c|c|}
\hline $\begin{array}{l}\text { Source of } \\
\text { Variation }\end{array}$ & $\mathrm{DF}$ & $\mathrm{y} 1$ & y2 & y3 & y4 & y5 & y6 & y7 & y8 & y9 & y10 & y11 & $\mathrm{y} 12$ \\
\hline Replication & 1 & 0.16 & $649.86^{*}$ & $9969.88^{*}$ & $24.74^{* *}$ & $172.25^{*}$ & 1.46 & 6.70 & 0.09 & $7,725.27$ & 1.62 & $188.57^{*}$ & $23,800.25^{* * *}$ \\
\hline Genotype(G) & 32 & $6.48^{* *}$ & $13137.07^{* *}$ & $1342.20^{*}$ & $10.75^{*}$ & $33.86^{* *}$ & $33.52^{* *}$ & $728.82^{* *}$ & $6.04^{* *}$ & $94,525.31^{* *}$ & $37.92^{* *}$ & $138.10^{* *}$ & $75,605.20^{* *}$ \\
\hline Year (Y) & 1 & $5.69^{* *}$ & $14757.92^{* *}$ & $1862.41^{* *}$ & $575.59^{* *}$ & $12244.18^{* * *}$ & 14.11 & $43803.66^{* *}$ & $162.17^{* *}$ & $74,05,314.47^{* *}$ & $45.63^{* *}$ & 16.26 & $9,14,497.22^{* *}$ \\
\hline Season $(\mathrm{S})$ & 2 & $6.68^{* *}$ & $41832.87^{* *}$ & $48681.06^{* *}$ & $24.47^{* *}$ & $5606.82^{* *}$ & $583.61^{* *}$ & $74258.39^{* *}$ & 3.07 & $59,76,917.48^{* *}$ & $461.71^{* *}$ & $322.47^{* *}$ & $16,01,497.21^{* *}$ \\
\hline $\mathbf{G} \times \mathbf{Y}$ & 32 & $0.54^{* *}$ & $953.79^{* *}$ & 731.89 & 7.23 & 23.17 & 3.36 & $266.52^{* *}$ & $1.88^{*}$ & $48,952.19^{* *}$ & $11.54^{* *}$ & $19.09^{* *}$ & $17,598.95^{* *}$ \\
\hline$G \times S$ & 64 & $0.62^{* *}$ & $1403.62^{* *}$ & 727.55 & 9.16 & 25.49 & 4.06 & $234.42^{* *}$ & $1.62^{*}$ & $41,449.75^{* *}$ & $20.81^{* *}$ & $30.13^{* *}$ & $9,300.83^{* *}$ \\
\hline $\mathbf{Y} \times \mathbf{S}$ & 2 & $1.93^{* *}$ & $10287.88^{* *}$ & 12013.25 & $188.63^{*}$ & $7386.70^{* *}$ & $439.27^{* *}$ & $18799.79^{* *}$ & $72.13^{* *}$ & $3,70,062 \cdot 10^{* *}$ & $252.96^{* *}$ & $216.57^{* *}$ & $1,67,873.95^{* *}$ \\
\hline $\mathbf{G} \times \mathbf{Y} \times \mathbf{S}$ & 64 & $0.55^{* *}$ & $1087.52^{* *}$ & 562.06 & 6.46 & $29.35^{* *}$ & 6.31 & & 1.05 & & $11.78^{* *}$ & $15.43^{*}$ & \\
\hline Error & 197 & 0.15 & 384.40 & 718.92 & 6.15 & 16.38 & 5.19 & 92.93 & 1.05 & $14,309.30$ & 3.81 & 10.03 & 535.44 \\
\hline Min & & 1.756 & 97.14 & 176.5 & 72.64 & 69.32 & 24 & 118 & 7 & 719 & 50.55 & 9.84 & 362 \\
\hline Max & & 4.236 & 215.4 & 224.25 & 76.59 & 76.35 & 30 & 147 & 10 & 1155 & 57.69 & 22.41 & 654 \\
\hline Std. range & & 0.826 & 0.765 & 0.237 & 0.053 & 0.095 & 0.225 & 0.219 & 0.352 & 0.465 & 0.133 & 0.771 & 0.590 \\
\hline C.V. $(\%)$ & & 31.43 & 29.23 & 16.28 & 4.17 & 14.76 & 13.25 & 21.00 & 18.08 & 30.35 & 7.17 & 32.68 & 30.14 \\
\hline \multicolumn{14}{|c|}{ y1: Fresh leaf weight $(\mathrm{g}) \quad$ y2: Leaf area $\left(\mathrm{cm}^{2}\right)$} \\
\hline \multicolumn{14}{|c|}{ y5: Moisture retention after $6 \mathrm{hrs}(\%)$ y6: Leaves per meter shoot $\quad$ y7: Length of longest shoot $(\mathrm{cm}) \quad$ y8: No of primary shoots } \\
\hline \multicolumn{4}{|c|}{ y9: Total shoot length (cm) } & \multicolumn{3}{|c|}{ y10: Leaf to shoot ratio (\%) } & \multicolumn{7}{|c|}{ y12: Leaf yield per plant (g) } \\
\hline
\end{tabular}


Table.2 Principal component analysis for leaf yield and quality traits in mulberry genotypes suitable for rainfed conditions

\begin{tabular}{|c|c|c|c|c|c|c|c|c|c|}
\hline Principal Component & PC1 & PC2 & PC3 & PC4 & PC5 & PC6 & PC7 & PC8 & PC9 \\
\hline Eigen values & 6.16 & 3.86 & 2.83 & 1.95 & 1.82 & 1.56 & 1.45 & 1.31 & 1.08 \\
\hline Proportion & 22.00 & 13.80 & 10.10 & 7.00 & 6.50 & 5.60 & 5.20 & 4.70 & 3.90 \\
\hline Cumulative Prop & 22.00 & 35.80 & 45.90 & 52.80 & 59.30 & 64.90 & 70.10 & 74.70 & 78.60 \\
\hline Fresh leaf weight (y1) & 0.378 & -0.039 & -0.09 & -0.008 & 0.048 & -0.02 & 0.026 & 0.159 & 0.080 \\
\hline Leaf area $(\mathrm{y} 2)$ & 0.374 & -0.032 & -0.106 & 0.009 & 0.079 & -0.028 & -0.003 & 0.103 & 0.064 \\
\hline Leaf moisture conten & 0.155 & 0.354 & -0.179 & 0.104 & -0.041 & -0.013 & -0.049 & 0.099 & -0.089 \\
\hline Moisture reteI & 0.182 & 0.116 & 0.293 & -0.314 & -0.064 & -0.055 & 0.174 & 0.142 & 0.198 \\
\hline Leaves per meter sh & -0.321 & 0.133 & 0.087 & 0.079 & -0.062 & 0.055 & 0.129 & -0.102 & 0.099 \\
\hline Length of the lo & 0.181 & 0.197 & -0.181 & -0.118 & 0.024 & -0.171 & 0.458 & -0.067 & -0.278 \\
\hline No of & 0.058 & 0.210 & 0.210 & 0.293 & -0.252 & 0.261 & -0.223 & -0.121 & 0.066 \\
\hline Total s & 0.176 & 0.327 & 0.157 & 0.172 & -0.210 & 0.049 & -0.136 & -0.171 & -0.072 \\
\hline Leaf $t$ & -0.069 & 0.205 & -0.279 & 0.177 & 0.119 & 0.022 & -0.085 & 0.429 & 0.225 \\
\hline Total & 0.134 & -0.155 & 0.007 & -0.005 & -0.126 & -0.441 & -0.077 & 0.295 & -0.034 \\
\hline Total sc & 0.03 & 0.122 & 0.293 & -0.412 & 0.110 & 0.038 & -0.188 & -0.043 & -0.298 \\
\hline Leaf & -0.143 & 0.121 & 0.295 & -0.115 & 0.106 & 0.156 & -0.075 & 0.220 & -0.224 \\
\hline Epicu & 0.062 & -0.065 & 0.074 & 0.243 & 0.407 & -0.139 & 0.152 & -0.214 & -0.023 \\
\hline Leaf ar & 0.309 & 0.109 & 0.112 & -0.057 & -0.042 & -0.003 & -0.087 & -0.186 & 0.194 \\
\hline Stom & -0.101 & 0.157 & -0.186 & -0.206 & 0.04 & -0.070 & -0.145 & -0.106 & 0.601 \\
\hline Lami & 0.068 & -0.130 & 0.288 & 0.226 & 0.011 & 0.181 & -0.039 & 0.488 & 0.027 \\
\hline Chlorophyll Content ind & 0.116 & -0.308 & 0.121 & 0.251 & -0.161 & 0.120 & 0.329 & -0.144 & 0.014 \\
\hline Relative & -0.034 & 0.115 & -0.270 & -0.067 & -0.130 & 0.435 & 0.067 & 0.206 & -0.072 \\
\hline Chlorophyll a/b ratio ( & 0.211 & 0.054 & -0.129 & -0.078 & 0.315 & 0.034 & -0.148 & -0.237 & -0.018 \\
\hline Water loss rate (y20) & 0.287 & -0.074 & -0.118 & -0.037 & 0.062 & 0.105 & -0.046 & 0.135 & -0.287 \\
\hline Speci & -0.108 & 0.364 & -0.107 & 0.135 & 0.060 & -0.014 & -0.080 & -0.130 & -0.292 \\
\hline Leaf fall at harvest (y22) & 0.293 & -0.138 & 0.048 & 0.154 & -0.147 & 0.093 & -0.292 & -0.104 & -0.006 \\
\hline Total soluble protein (y23) & 0.029 & 0.028 & 0.181 & -0.035 & 0.592 & 0.121 & -0.208 & 0.070 & 0.085 \\
\hline Bacterial leaf spot (y24) & -0.05 & -0.152 & -0.240 & 0.362 & 0.252 & 0.074 & -0.124 & -0.073 & -0.099 \\
\hline Pseudocercospora leaf spot (y2 & -0.053 & -0.146 & -0.367 & -0.267 & -0.196 & 0.069 & -0.283 & 0.016 & -0.182 \\
\hline Myrothecium leaf spot (y26) & 0.124 & -0.285 & -0.065 & -0.176 & -0.092 & 0.196 & -0.203 & -0.217 & 0.063 \\
\hline ifestation (y27) & -0.094 & 0.010 & 0.079 & 0.161 & -0.129 & -0.571 & -0.379 & 0.036 & -0.118 \\
\hline Leaf yield per plant (y28) & 0.250 & 0.322 & -0.015 & 0.09 & -0.043 & -0.048 & 0.110 & 0.012 & 0.115 \\
\hline
\end{tabular}


Table.3 PCA scores of mulberry genotypes suitable for rainfed conditions

\begin{tabular}{|c|c|c|c|c|c|c|c|c|c|c|}
\hline \multirow[t]{2}{*}{ Genotype } & \multicolumn{9}{|c|}{ Principle components } & \multirow[t]{2}{*}{ Rank } \\
\hline & 1 & 2 & $\mathrm{C} 3$ & $\mathrm{DCA}$ & PC5 & PC6 & $\mathrm{C} 7$ & 8 & PC9 & \\
\hline & 1.013 & 2.948 & 270 & -0.004 & 1.015 & -0.164 & 0.461 & .537 & 510 & 4 \\
\hline & & & 253 & & 1.084 & 0.848 & -0.569 & -0.812 & -1.493 & 12 \\
\hline & .677 & 3.112 & -0.773 & -0.500 & -0.374 & 0.397 & 0.059 & 0.157 & -0.060 & 14 \\
\hline & 0.813 & 2.267 & -0.156 & -1.235 & -0.158 & -0.222 & -0.633 & 0.681 & 0.767 & 9 \\
\hline PYD 05 & -1.252 & -1.561 & -4.647 & 2.002 & 2.894 & 2.009 & -0.290 & -0.539 & -0.321 & 28 \\
\hline & .357 & 0.071 & -0.604 & -0.989 & 1.990 & -2.339 & 0.244 & 1.176 & -0.617 & 16 \\
\hline & -0.143 & 1.453 & 729 & 0.24 & 0.286 & -1.236 & 1.793 & -0.996 & (1) & 5 \\
\hline & 5.321 & 2.210 & 715 & -0.026 & 0.887 & -0.386 & -0.891 & -1.970 & 0.756 & 1 \\
\hline PYD 09 & 3.545 & 0.714 & .908 & -1.004 & 1.455 & -1.829 & -0.951 & -0.083 & -0.303 & 10 \\
\hline PYD 10 & 2.969 & -4.730 & 3.576 & -0.284 & 1.925 & -0.285 & -0.774 & -0.309 & 1.198 & 26 \\
\hline & 1.577 & -3.478 & -0.271 & -0.036 & -0.635 & 0.020 & -0.254 & -1.458 & 0.910 & 30 \\
\hline & -1.941 & & 2.284 & 0.843 & -0.102 & 0.743 & 0.880 & & 1.523 & 21 \\
\hline PYI & & & -0.803 & -0.223 & 0.666 & -2.983 & 0.929 & -0.734 & -1.953 & 27 \\
\hline PYD 14 & -1.841 & -0.491 & 0.873 & 1.876 & 2.036 & 0.110 & -0.267 & -0.650 & -0.324 & 25 \\
\hline & -0.271 & 1.602 & 1.200 & 1.116 & 0.749 & 1.937 & 1.783 & 0.711 & 0.462 & 2 \\
\hline PYD 16 & 6 & 0 & 92 & .363 & -0.317 & -1.126 & 2.330 & -0.693 & -0.73 & 6 \\
\hline & & & 5 & 0.958 & 0.040 & -0.627 & 0.154 & 1.490 & 0.663 & 22 \\
\hline & -3.572 & & 4 & $-2 .($ & -0.204 & 0.720 & -2.210 & 1.376 & -0.5 & 24 \\
\hline & -3.666 & & & -2.446 & -0. & & -0.660 & -1.611 & 0. & 29 \\
\hline & -3.320 & & 347 & 1.811 & -2.945 & -2.007 & -0.549 & -1.305 & -0.665 & 33 \\
\hline PYD 21 & 1.705 & 3.255 & -0.257 & 0.399 & -0.051 & 0.231 & -2.467 & 0.056 & 0.850 & 20 \\
\hline & & & -3.452 & -3.295 & -0.234 & -1.108 & -0.554 & 0.410 & 0.784 & 32 \\
\hline & & & & & & 1.257 & 1.756 & 0.036 & -0.179 & 18 \\
\hline & & & -1.043 & 0.897 & -2.233 & 1.591 & -0.883 & -2.469 & -1.707 & 13 \\
\hline PYD 25 & -2.462 & -2.003 & -2.297 & 1.185 & 0.059 & 0.078 & -0.307 & -0.804 & 0.973 & 31 \\
\hline & & & -0.168 & -2.002 & -0.484 & 0.392 & 1.672 & -0.108 & -0.847 & 11 \\
\hline & & & -1.605 & 1.765 & -2.051 & -1.323 & 0.742 & 1.337 & 2.551 & 3 \\
\hline & 0.257 & 1.429 & 0.303 & -2.014 & -0.114 & 1.019 & 1.103 & -0.347 & 0.411 & 7 \\
\hline PYD 29 & -1.052 & & -0.252 & 0.106 & -0.427 & 1.490 & 1.753 & 1.696 & -0.599 & 23 \\
\hline & & -0.521 & -1.610 & 0.386 & 1.553 & 1.152 & 0.590 & 0.294 & 0.812 & 17 \\
\hline C-1730 & 1.524 & -0.872 & 0.626 & 0.959 & -0.749 & 0.801 & -1.578 & 1.771 & -1.207 & 19 \\
\hline & 3.292 & -1.568 & 0.051 & 1.674 & -1.804 & 0.214 & -1.209 & 2.022 & -1.255 & 15 \\
\hline C-2038 & 4.170 & -0.087 & 0.226 & 0.564 & -0.964 & -0.72 & -1.2 & 0.264 & 0.931 & 8 \\
\hline
\end{tabular}


Table.4 AMMI analysis of mulberry genotypes over seasons for leaf yield and its component traits

\begin{tabular}{|l|c|c|c|c|c|c|}
\hline \multicolumn{1}{|c|}{ Source of Variation } & DF & FLW & TSL & LSR & LYP & $\%$ SS \\
\hline REP(ENV) & 3 & 0.53 & 31241 & 12.38 & 13737 & 0.80 \\
\hline Genotype(G) & 32 & $6.48^{* *}$ & $94518^{* *}$ & $37.90^{* *}$ & $75605^{* *}$ & 4.40 \\
\hline Environment/Season(E) & 2 & $6.68^{*}$ & $5976799^{* *}$ & $461.45^{* *}$ & $1601497^{* *}$ & 93.22 \\
\hline G $\times$ E & 64 & $0.62^{* *}$ & 41454 & $20.82^{* *}$ & 9301 & 0.54 \\
\hline IPCA 1 & 33 & 0.33 & 20874 & 11.91 & 5410 & 0.31 \\
\hline IPCA 2 & 31 & 0.28 & 20569 & 8.81 & 3841 & 0.22 \\
\hline Residuals & 294 & 0.31 & 48356 & 8.13 & 8601 & 0.50 \\
\hline $\begin{array}{l}\text { FLW: Fresh leaf weight (g); } \\
\text { shoot ratio (\%); } \text { : LYP: Leaf yield per plant (g) }\end{array}$
\end{tabular}

$*$, ** Significant at $5 \%$ \& $1 \%$ level of significance, respectively

Table.5 Comparison of mulberry genotypes suitable for rainfed conditions on seasonal performance, AMMI Stability values and annual yield

\begin{tabular}{|c|c|c|c|c|c|c|c|c|c|c|c|}
\hline \multirow[t]{2}{*}{ Name } & \multicolumn{5}{|c|}{ Seasonal Performance } & \multicolumn{4}{|c|}{ AMMI Stability } & \multicolumn{2}{|c|}{ Annual Leaf Yield } \\
\hline & $\mathrm{S} 1$ & S2 & S3 & Mean & Rank & IPC1 & IPC2 & ASV & Rank & $\mathrm{kg} / \mathrm{yr}$ & $\begin{array}{c}\% \text { more overC- } \\
2038\end{array}$ \\
\hline PYD 01 & 719 & 665 & 469 & 618 & 3 & -1.74 & 0.67 & 2.25 & 5 & $1.852^{*}$ & 16 \\
\hline PYD 02 & 552 & 676 & 399 & 542 & 10 & 3.98 & 5.70 & 7.53 & 31 & 1.624 & \\
\hline PYD 03 & 595 & 550 & 319 & 488 & 17 & -2.44 & 2.18 & 3.72 & 19 & 1.463 & \\
\hline PYD 04 & 670 & 621 & 453 & 581 & 5 & -0.63 & -0.33 & 0.85 & 3 & $1.742^{*}$ & 9 \\
\hline PYD 05 & 521 & 510 & 366 & 466 & 21 & 1.88 & -0.89 & 2.49 & 9 & 1.395 & \\
\hline PYD 06 & 561 & 529 & 371 & 487 & 18 & 0.45 & -0.56 & 0.79 & 2 & 1.459 & \\
\hline PYD 07 & 713 & 579 & 433 & 575 & 7 & -3.90 & -2.09 & 5.25 & 24 & $1.725^{*}$ & 8 \\
\hline PYD 08 & 770 & 711 & 480 & 654 & 1 & -3.06 & 1.99 & 4.27 & 20 & $1.960^{*}$ & 23 \\
\hline PYD 09 & 619 & 693 & 396 & 569 & 8 & 1.05 & 5.95 & 6.09 & 27 & $1.706^{*}$ & 7 \\
\hline PYD 10 & 481 & 520 & 331 & 444 & 23 & 2.78 & 1.35 & 3.69 & 18 & 1.331 & \\
\hline PYD 11 & 608 & 488 & 326 & 474 & 20 & -3.75 & -1.34 & 4.82 & 22 & 1.421 & \\
\hline PYD 12 & 640 & 490 & 360 & 497 & 16 & -4.14 & -2.92 & 5.89 & 25 & 1.489 & \\
\hline PYD 13 & 394 & 433 & 355 & 394 & 30 & 6.24 & -3.01 & 8.28 & 33 & 1.181 & \\
\hline PYD 14 & 416 & 438 & 360 & 405 & 28 & 5.47 & -3.19 & 7.48 & 29 & 1.212 & \\
\hline PYD 15 & 653 & 576 & 445 & 558 & 9 & -0.78 & -2.09 & 2.30 & 6 & $1.674^{*}$ & 5 \\
\hline PYD 16 & 640 & 567 & 376 & 528 & 13 & -2.45 & 0.29 & 3.04 & 14 & 1.581 & \\
\hline PYD 17 & 455 & 461 & 227 & 381 & 31 & -0.17 & 2.80 & 2.81 & 11 & 1.141 & \\
\hline PYD 18 & 391 & 411 & 286 & 362 & 33 & 3.88 & -1.33 & 4.98 & 23 & 1.086 & \\
\hline PYD 19 & 521 & 365 & 249 & 378 & 32 & -3.96 & -3.48 & 6.01 & 26 & 1.133 & \\
\hline PYD 20 & 427 & 479 & 383 & 429 & 26 & 6.29 & -2.16 & 8.07 & 32 & 1.286 & \\
\hline PYD 21 & 668 & 637 & 478 & 594 & 4 & 0.48 & -0.52 & 0.79 & 1 & $1.783^{*}$ & 11 \\
\hline PYD 22 & 500 & 410 & 291 & 400 & 29 & -1.00 & -2.71 & 2.98 & 13 & 1.198 & \\
\hline
\end{tabular}




\begin{tabular}{|l|l|l|l|l|c|c|c|c|c|c|c|}
\hline PYD 23 & 611 & 548 & 435 & 531 & 12 & 0.42 & -2.66 & 2.71 & 10 & 1.594 & \\
\hline PYD 24 & 555 & 571 & 386 & 504 & 15 & 1.84 & 1.00 & 2.48 & 8 & 1.510 & \\
\hline PYD 25 & 571 & 396 & 254 & 407 & 27 & -5.68 & -2.69 & 7.52 & 30 & 1.220 & \\
\hline PYD 26 & 650 & 589 & 496 & 578 & 6 & 1.13 & -3.41 & 3.68 & 17 & $1.733^{*}$ & 8 \\
\hline PYD 27 & 720 & 748 & 437 & 635 & 2 & -1.54 & 6.04 & 6.33 & 28 & $1.904^{*}$ & 19 \\
\hline PYD 28 & 511 & 501 & 345 & 452 & 22 & 1.53 & -0.42 & 1.94 & 4 & 1.355 & \\
\hline PYD 29 & 511 & 472 & 346 & 443 & 24 & 1.15 & -1.88 & 2.36 & 7 & 1.328 & \\
\hline PYD 30 & 565 & 494 & 269 & 443 & 25 & -3.43 & 1.65 & 4.55 & 21 & 1.327 & \\
\hline C-1730 & 536 & 558 & 332 & 475 & 19 & 0.85 & 2.65 & 2.85 & 12 & 1.423 & \\
\hline S-1635 & 569 & 590 & 360 & 506 & 14 & -1.45 & 2.62 & 3.17 & 15 & 1.518 & \\
\hline C-2038 & 626 & 606 & 369 & 534 & 11 & -1.45 & 2.62 & 3.17 & 16 & 1.600 & \\
\hline
\end{tabular}

Table.6 Leaf yield and growth traits of top ranking genotypes suitable for rainfed conditions

\begin{tabular}{|c|c|c|c|c|c|c|c|c|c|c|c|c|}
\hline Name & $\mathbf{y 1}$ & $\mathbf{y ~ 2}$ & $\mathbf{y ~ 3}$ & $\mathbf{y 4}$ & $\mathbf{y 5}$ & $\mathbf{y 6}$ & $\mathbf{y 7}$ & $\mathbf{y 8}$ & $\mathbf{y 9}$ & $\mathbf{y 1 0}$ & $\mathbf{y 1 1}$ & $\mathbf{y 1 2}$ \\
\hline PYD 08 & 4.144 & 206.82 & 194.56 & 74.96 & 75.69 & 24 & $139^{*}$ & $10^{*}$ & $1155^{*}$ & $54.21^{*}$ & $19.26^{*}$ & $654^{*}$ \\
\hline PYD 15 & 3.278 & 167.99 & 204.84 & 75.05 & 74.18 & $29^{*}$ & 134 & 9 & 970 & $55.64^{*}$ & $15.08^{*}$ & $558^{*}$ \\
\hline PYD 27 & 3.351 & 163.66 & 200.14 & 75.29 & 73.92 & $27^{*}$ & $139^{*}$ & 9 & 1025 & $57.07^{*}$ & $17.43^{*}$ & $635^{*}$ \\
\hline PYD 01 & 3.164 & 159.16 & 208.27 & 76.59 & 74.41 & 26 & $140^{*}$ & 8 & 955 & $54.40^{*}$ & $14.17^{*}$ & $618^{*}$ \\
\hline PYD 07 & 2.633 & 136.40 & 205.53 & 75.28 & 74.72 & $29^{*}$ & $140^{*}$ & 8 & 988 & $54.21^{*}$ & $13.19^{*}$ & $575^{*}$ \\
\hline C-2038 & 4.433 & 222.43 & 199.62 & 75.13 & 74.70 & 24 & 129 & 9 & 1026 & 52.54 & 22.92 & 534 \\
\hline CD 5\% & 0.311 & 15.78 & 21.58 & 1.99 & 3.26 & 2 & 8 & 1 & 96 & 1.57 & 2.55 & 19 \\
\hline
\end{tabular}

$\begin{array}{llc}\text { y1: Fresh leaf weight }(\mathrm{g}) & \text { y2: Leaf area }\left(\mathrm{cm}^{2}\right) & \text { y3: Specific leaf area }\left(\mathrm{cm}^{2} \mathrm{~g}^{-1}\right) \\ \text { y4: Leaf moisture content }(\%) & \text { y5: Moisture retention aft 6hrs }(\%) \text { y6: Leaves per mt. shoot }(\text { No. }) \\ \begin{array}{lll}\text { y7 : Length of longest shoot }(\mathrm{cm}) & \text { y8: No of primary shoots } & \text { y9: Total shoot length }(\mathrm{cm}) \\ \text { y10: Leaf to shoot ratio }(\%) & \text { y11: Leaf fall at harvest }(\%) & \text { y12: Leaf yield per plant }(\mathrm{g})\end{array}\end{array}$

Table.7 Leaf quality characteristics and pest-disease severity of top ranking genotypes

\begin{tabular}{|c|c|c|c|c|c|c|c|c|c|c|c|c|c|c|c|c|}
\hline Name & $\mathbf{y 1 3}$ & $\mathbf{y 1 4}$ & $\mathbf{y 1 5}$ & $\mathbf{y 1 6}$ & $\mathbf{y 1 7}$ & $\mathbf{y 1 8}$ & $\mathbf{y 1 9}$ & $\mathbf{y 2 0}$ & $\mathbf{y 2 1}$ & $\mathbf{y 2 2}$ & $\mathbf{y 2 3}$ & $\mathbf{y 2 4}$ & $\mathbf{y 2 5}$ & $\mathbf{y 2 6}$ & $\mathbf{y 2 7}$ & $\mathbf{y 2 8}$ \\
\hline PYD 08 & 2.39 & 34.29 & $49.22^{*}$ & 283 & $346^{*}$ & 3.60 & 1053 & 132 & 16.28 & 79.02 & 476 & $3.73^{*}$ & 4.14 & 5.98 & 5.26 & 29.54 \\
\hline PYD 15 & 2.03 & 31.26 & 42.86 & $1675^{*}$ & $336^{*}$ & 1.85 & $934^{*}$ & $155^{*}$ & 18.25 & 80.71 & $274^{*}$ & $2.74^{*}$ & 5.38 & 4.94 & 3.62 & 8.17 \\
\hline PYD 27 & 2.68 & 25.84 & 31.49 & 643 & $329^{*}$ & 2.94 & 1346 & $143^{*}$ & 15.53 & $86.52^{*}$ & $324^{*}$ & 2.20 & 4.34 & 7.85 & 2.61 & 35.60 \\
\hline PYD 01 & 2.03 & 35.35 & $46.39^{*}$ & $1168^{*}$ & $332^{*}$ & 2.91 & $786^{*}$ & $151^{*}$ & 13.88 & $85.53^{*}$ & 455 & $2.52^{*}$ & 4.76 & 8.58 & 1.83 & 36.28 \\
\hline PYD 07 & 2.45 & 33.35 & $46.48^{*}$ & 358 & $338^{*}$ & 2.91 & $975^{*}$ & 135 & 18.22 & 77.14 & $306^{*}$ & 2.23 & 5.85 & 4.88 & 4.26 & 26.78 \\
\hline C-2038 & 2.89 & 35.25 & 42.66 & 496 & 319 & 3.90 & 1096 & 136 & 18.59 & 80.02 & 478 & 2.27 & 8.00 & 7.94 & 3.52 & 30.05 \\
\hline Min & 1.81 & 23.2 & 31.49 & 283 & 312 & 1.2 & 648 & 124 & 11.37 & 65.53 & 209 & 1.37 & 2.97 & 0.00 & 1.83 & 8.17 \\
\hline Max & 2.81 & 43.65 & 49.88 & 2344 & 346 & 3.6 & 1434 & 162 & 20.18 & 91.95 & 610 & 3.73 & 17.04 & 19.27 & 8.72 & 55.75 \\
\hline Std. range & 0.417 & 0.611 & 0.426 & 2.476 & 0.103 & 1.027 & 0.810 & 0.275 & 0.527 & 0.322 & 1.058 & 0.970 & 2.163 & 1.926 & 1.444 & 1.660 \\
\hline CD 5\% & 0.10 & 1.54 & 1.53 & 168 & 3 & 0.24 & 66 & 4 & 0.83 & 1.91 & 36 & 0.10 & 0.78 & 1.35 & 0.43 & 2.66 \\
\hline
\end{tabular}

y13 : Total chlorophyll $\left(\mathrm{mg} \mathrm{g}^{-1}\right) \quad$ y14: Total Soluble protein $\left(\mathrm{mg} \mathrm{g}^{-1}\right)$ y15: Total Soluble sugars $\left(\mathrm{mg} \mathrm{g}^{-1}\right)$

y16: Leaf Proline content $(\mu \mathrm{g} / \mathrm{g})$ y17: Epicuticular wax $\left(\mu \mathrm{g} / \mathrm{cm}^{2}\right) \quad$ y18: Leaf area index

y19: Stomata frequency $\left(\mathrm{No} / \mathrm{mm}^{2}\right)$ y20: Leaf thickness $(\mu \mathrm{m}) \quad$ y21: Chlorophyll content Index

y22: Relative water content (\%) y23: Water loss rate $\left(\mathrm{mg} \mathrm{g}^{-1} \mathrm{~h}^{-1}\right) \quad$ y24: Chlorophyll a / b ratio 
Fig.1 Biplot analysis based on PC1 and PC2 for mulberry leaf yield and quality traits

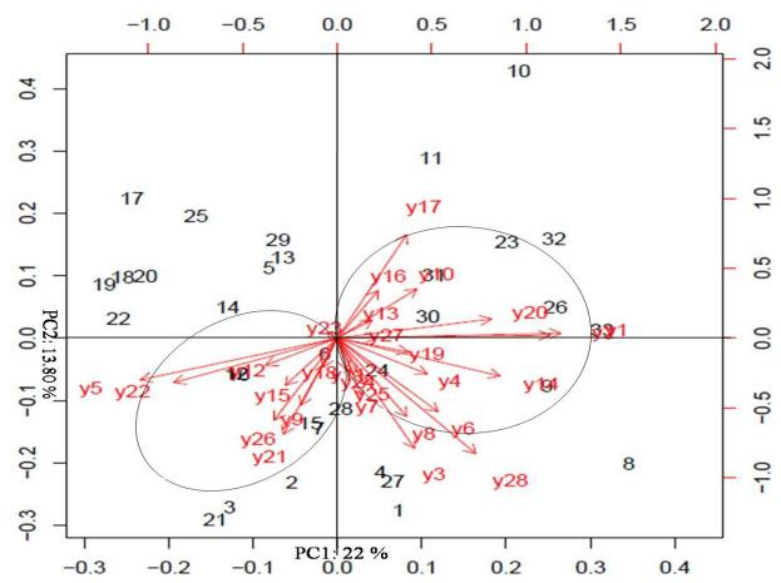

Fig.2 Biplot based on AMMI-II model for leaf yield across seasons

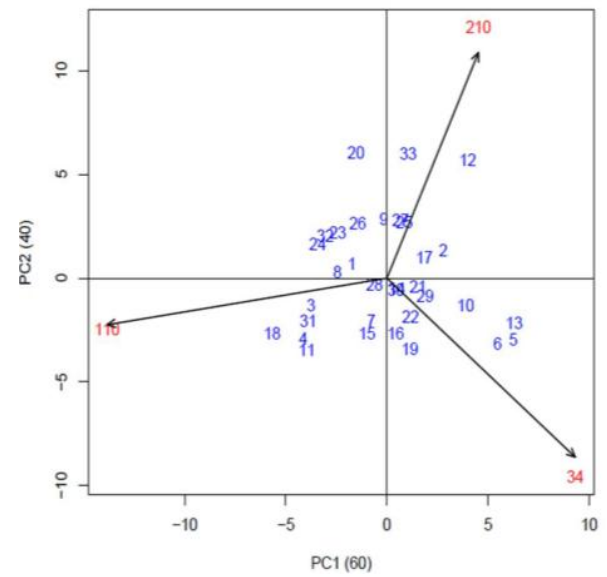

Note: 110: July Season (S1); 210: September season (S2); 34: November season (S1); 1 to 30 denotes PYD 1 to 30, C-1730:31; S-1635: 32 and C-2038: 33

Genotypes PYD 21(3.255), PYD 03(3.112), PYD 01(2.948), PYD 02(2.757), PYD 04 (2.267) and PYD 28 (1.429) had highest score in PC2. They exhibited high value for specific leaf area, leaf moisture, leaves per meter shoot, total shoots length and leaf yield per plant.

Genotype PYD 10 (3.576), PYD 16 (2.392), PYD 12 (2.284), PYD 18 (2.174), PYD 19 (2.094) and PYD 17 (1.505) showed highest score in PC3 and exhibited higher values for moisture retention capacity, total soluble sugars and leaf proline content. In PC4, PYD
25 (1.185) and PYD 20 (1.811) recorded the highest PC score indicating that they had high value for shoots per plant, Bacterial leaf spot and tukra infestation. PYD 05(2.894) exhibited the highest PC score followed by PYD 14 (2.036), PYD 06 (1.990) and PYD 30 (1.553) in PC5 indicating total soluble protein, epicuticular wax content, chlorophyll $\mathrm{a} / \mathrm{b}$ ratio were of high values. In PC6, PYD 15 (1.937) and PYD 24 (1.591) found to have highest PC score and have higher value for relative water content, Pseudocercospora leaf spot and Myrothecium leaf spot. PYD 07 (1.793), PYD 29 (1.753), PYD 13 (0.929) in 
PC7 and only C-1730 (1.771) in PC8 exhibited high values for longest shoot, chlorophyll content index, and leaf to shoot ratio, total chlorophyll content, lamina thickness, respectively. In PC9, PYD 27 (2.551) and PYD 22 (0.784) recorded highest scores indicating that they had high value for stomata frequency. Similar studies were also conducted by Salcedo et al., (2017) and Chanotra et al., (2019).

Several selection indices have been used as an effective selection criterion in plant breeding programs for different crops (Dolan et al., 1996; Xie et al., 1998 and Vikram and Roy, 2003; Xie et al., 1998). The PC scores can be utilized to construct precise selection index whose intensity can be decided by variability explained by each of principal component. PCA index for each genotype is computed and ranks were assigned. Among the 30 genotypes evaluated, seven genotypes such as PYD 08, PYD 15, PYD 27, PYD 01, PYD 07, PYD 16 and PYD 28 were top rankers compared to variety C-2038. Jha et al., (2016) performed PCA for identification and ranking of advanced genotypes of soybean based on combination of various phenotypic traits.

\section{AMMI analysis of mulberry genotypes across seasons}

The AMMI method was used for analysis of stability across seasons among the genotypes studied. ANOVA for four traits across three seasons over two years was analyzed according to the AMMI model is presented in table 4. The ANOVA indicated significant differences $(\mathrm{P}<0.01)$ for seasons $(\mathrm{E})$, varieties (G) and $G \times$ E interaction for fresh leaf weight and leaf to shoot ratio. Leaf yield was significantly affected by the environment and explained $93.2 \%$ of the total variation, while $\mathrm{G} \times \mathrm{E}$ interaction and genotype effects captured only $0.5 \%$ and $4.4 \%$ variation, respectively. The large sum of squares and significant effect for seasons demonstrated that the experiments were carried out under divergent climatic condition. Similar results were observed in peanuts, in which most of the variation (93\%) was attributable to environmental factors demonstrating that selecting the best genotypes is not an easy task (Oliveira and Godoy, 2006). The performance of genotypes averaged over three seasons for two years is presented in Table 5 which depict that PYD 08 and PYD 18 had the highest $(654 \mathrm{~g})$ and the lowest $(362 \mathrm{~g})$ leaf yield per plant, respectively. Although the genotypes showed less consistent performances across all seasons, PYD 08 (654g), PYD 27 (635g), PYD 01 (618g) were the top performers, while PYD 18, PYD 19, PYD 17, PYD 13, PYD 22 were the poorest and remaining were moderate yielders. The mean leaf yield of all the genotypes ranged from 770g in July (S1) to 227g in Nov season (S3). The average productivity of all seasons for all the genotypes was $495 \mathrm{~g}$. On the basis of environmental index value considering negative and positive, Nov season was found to be poor, September was medium and July was the most favorable environment.

Biplot analysis is possibly the most powerful interpretive tool for AMMI models, where scores for IPCA1 and IPCA2 are plotted. The variance of GEI was partitioned among the first two interaction principle component axis (IPCA). IPCA 1 and IPCA 2 axes explained $0.31 \%$ and $0.22 \%$ of the total GEI and both were non-significant $(\mathrm{P}<0.01)$. According to Duarte and Vencovsky (1999), biplot analysis revealed stable environments and genotypes that were located near the origin with low scores for the two axes of the interaction. The varieties and environments those were farthest from the origin being more responsive. Accordingly, September season (E2) had short spokes and did not exert strong interactive force while environments, July season (E1) having long spokes exerted 
strong interaction. Genotypes, PYD 13, PYD 05, PYD 06, PYD 12, PYD 33 and PYD 20 with high IPCA score and away from origin were most responsive. Genotypes, PYD 14, PYD 28, PYD 30, PYD 21, PYD 01, PYD 08, PYD 07, PYD 21, PYD 17 and PYD 22 were close to the origin, indicating minimal interaction of these genotypes with environments and were most stable performers across the three seasons. The remaining genotypes were scattered away from the origin in the Biplot indicating that the varieties were moderately sensitive to environmental interactive forces. Interaction of varieties with specific environmental conditions was judged by projection of variety points on to environment spokes. Several authors reported and attributed most of the $\mathrm{G} \times \mathrm{E}$ interaction sum squares to first two IPCA axes (Crossa et al., 1990 and Purchase et al., 2000). Several studies have been conducted on stability performance for leaf yield of mulberry (Ghosh et al., 2013; Bhavyashree et al., 2015).

AMMI analysis does not provide a quantitative measure of stability. For this reason, Purchase et al., (2000) proposed an ASV measure to quantify and classify genotypes according to their yield stability wherein ASV is the distance of the varieties from point zero of the scatter diagram (IPCA1 vs. IPCA2). The AMMI model IPCA1 and IPCA2 scores of leaf yield for each genotype and the corresponding AMMI stability value (ASV) are shown in Table 5. According to ASV ranking, genotype PYD 21, PYD 06, PYD 04, PYD 28 and PYD 01 had the lowest value and were the most stable genotypes, while genotype PYD 13 and PYD 20 were relatively less stable. The ASV parameter has been used as an auxiliary criterion to define more stable genotypes in other crops such as wheat (Farshadfar et al., 2011) and rice (Das et al., 2010).
In conclusion, Mulberry leaf yield is a complex trait that is influenced by a number of component characters along with the environment directly or indirectly. The traits such as leaf area, leaf moisture, moisture retention capacity, length of longest shoot, primary shoots per plant, total shoots length, total soluble sugars, leaf area index and chlorophyll $\mathrm{a} / \mathrm{b}$ ratio are important yield contributing traits under rainfed condition. The genotypes PYD 08, PYD 15, PYD 27, PYD 01 and PYD 07 were preliminarily identified as superior over C2038 with 5 $23 \%$ yield advantage and better stability across seasons.

\section{References}

Annual report, 2018-2019.Central Silk Board, Mininstry of textiles, Government of India. Page no. 95. www.csb.org.in/201819

Arnon, D.I., 1949.Copper enzymes in intact chloroplast. Polyphenolyxidase in Beta vulgaris. Plant Physiol., 24:1-15.

Ashiru, M. O. 2002. The effect of mulberry varieties on the performance of Chul Thai-5 silkworm race. Discovery and Innovation, 14, 77-83.

Banerjee, R., Roychowdhuri, S., Sau, H., Das, B. K., Ghosh, P. and Saratchandra, B. 2007.Genetic diversity and interrelationship among mulberry genotypes. J. Genet. Genomics. 34(8):691-97.

Banerjee, R., Roychowdhuri, S., Sau, H., Das, B. K., Saha, A. K, Saratchandra B. and Bajpai A. K. 2011. Phenotypic divergence in mulberry (Morus spp.) germplasm collections. $J$. Crop Improv., 25: 459-471.

Barrs, H. D. and Weatherley, P. E. 1962.A reexamination of the relative turgidity technique for estimating water deficit in leaves. Aust. J. Biol. Sci., 15:413-428.

Bates L. S., Waldren R. P. and Teare, I. D. 1973. Rapid determination of free proline for water stress studies. Plant Soil, 39: 
205-207.

Bhavyashree, S., Krishnamurthy, K. N., and Chikkalingaiah, 2015. Identification of Stable Genotypes of Mulberry using Some Parametric Methods. Mysore J. Agric. Sci., 49 (3): 492-496.

Biradar, J., Chikkalingaiah, Banuprakash, K. G., Narayanaswamy, T. K. and Bhaskar, R. N., 2015. Estimation of genetic variability and correlation studies in mulberry Morus spp. Mysore J. Agric., Sci., 49(3):516-522.

Cao, X., Shen, Q., Shang, C. Yang, H., Liu, L. and Cheng, J. 2019. Determinants of shoot biomass production in mulberry: combined selection with leaf morphological and physiological traits. Plants (Basel, Switzerland). 8(118):1-29.

Chaluvachari and Bongale, U. D. 1994. Leaf quality evaluation of selected mulberry genotypes by biochemical and bioassay studies. Proceedings of the Fourth All India Conference on Cytology and Genetics held at Karnataka State Sericulture Research \& Development Institute, Thalaghattapura, Bangalore, Karnataka, India, pp.121-124.

Chanotra, S., Bali, R. K., and Bali. K. 2019. Estimation of Genetic Variability and Heritability in Selected Mulberry Germplasm Accessions (Morus spp.). Int. J. Curr. Microbiol. App. Sci., 8(02): 493499.

Crossa, J., Gauch, H. G., Zobel, R.W. 1990. Additive main effect and multiplicative interaction analysis of two international maize cultivar trials. Crop Sci., 30: 493500.

Das, S., Misra, R. C., Patnaik, M. C., Das, S. R. 2010. G×E interaction, adaptability and yield stability of mid-early rice genotypes. The Indian J. Agri. Res., 44: 104-111.

Das, T. R. and Baisakh, B. 2019. Selection indices and discriminant function analysis for grain yield in greengram [Vignaradiata (L.) Wilczek].e-planet, 17 (1): 13-21.

Dolan, D. J., Stuthman, D. D., Kolb, F. L. and
Hewings, A. D. 1996.Multiple traits selection in a recurrent selection population in Oat (Avena sativa L.). Crop Sci., 36(5): 1207-1211.

Doss, S. G., Chakraborti, S. P., Roychowdhuri, S., Das, N. K., Vijayan, K. Ghosh, P. D., Rajan, M, V. and Qadri, S. M. H., 2012. Variability, heritability and genetic advance in mulberry (Morus spp.) for growth and yield attributes. J. Agri. Sci., 3:208-213.

Doss, S. G., Rahman, M. S., Debnath, S., Ghosh, M. K., Sau, H., Ghosh, P. L. and Sarkar, A. 2006. Variability, heritability and genetic advance in nine germplasm lines of mulberry (Morus spp.). Indian J. Genet., 66:169-170.

Duarte, J. B. and Vencovsky, R. 1999. Genotype $\times$ environment interaction: an introduction to AMMI analysis = Interaçãogenótipos $\times$ ambientes: umaintrodução à análise AMMI. SociedadeBrasileira de Genética, RibeirãoPreto, SP, Brazil (in Portuguese).

Dubey, N., Avinashe, HA, and Shrivastava, A. N. 2018. Principal component analysis in advanced genotypes of soybean [Glycine $\max (1$.$) merrill] over seasons. Plant$ Archi., 18 (1): 501-506.

Ebercon, A., Blum, A. and Jordan, W. R. 1977. A rapid colorimetric method for epicuticular wax content of sorghum leaves. Crop Sci., 17: 179-180.

Falconer, D.S. and Mackay, T. F. C. 1996. Introduction to Quantitative Genetics. 4ed. London: Longman Scientific and Technical, Essex, England.

Farshadfar, E., Mahmodi, N. and Yaghotipoor, A. 2011. AMMI stability value and simultaneous estimation of yield and yield stability y in bread wheat (Triticum aestivum L.). Australian J. Crop Sci.,5(13): 1837-1844.

Fonseca,T. C., Almeida, J. E. and Fonseca, A. S. 1990. Effect of mulberry selection on silkworm feeding. Sericologia, 30(4):475477.

Fotadar, R. K., Ahsan, M. M., Dhar, K. L. and Bhakuni, B. S. 1989., Evaluation and 
utilization of genetic variability in mulberry. Indian J. Seric., 28(2):150-158.

Gabriel, K. R. 1971. The biplot-graphical display of matrices with applications to principal component analysis. Biometrika, 58:453-467.

Gauch, H. G, and Zobel, R. W. 1996. AMMI analysis of yield trials. In: Kang M. S., Gauch, H. G. (eds) Genotype by environment interaction. CRC Press. Boca Raton, FL.

Gauch, H.G., and Zobel. R. W. 1997. Identifying mega-environments and targeting genotypes. Crop Science 37:311-326.

Ghosh, M. K. Bindroo, B. B., Das, N. K., and Singh, M. K. 2013. Yield stability in mulberry over different regions of Eastern and North-Eastern India. J. Crop Weed, 9(1):103-105.

Harman, H.H. 1976. Modern factor analysis. 3nd Ed. University of Chicago Press, Chicago, pp. 376.

Horie, Y. and H. Watanabe, 1980. Recent advances in sericulture. Annu. Rev. Entomol., 25: 9-71.

Jha, A., A. N. Shrivastava and S. Mishra, 2016.Principal component analysis in advanced genotypes of soybean (Glycine $\max$ (L.)Merrill)" during Kharif-2014. Adv. Life Sci., 5(9): 3508-3513.

Jolliffe, I. T. 1986. Principal Component Analysis and Factor Analysis. In: Principal Component Analysis, Springer, New York, 115-128.

Kang, M.S. 1998. Using genotype-byenvironment interaction for crop cultivar development. Advance Agr., 62: 199-253.

Kitajima, Kaoru \& Hogan, K..2003. Increases of chlorophyll $\mathrm{a} / \mathrm{b}$ ratios during acclimation of tropical woody seedlings to nitrogen limitation and high light. Plant, cell \& envi., 26. 857-865.

Lowry, O.H., Roserbrough, N.J., Farr, A. L and Randall, R. J. 1951.Protein measurement with tliefoliri phenol reagent. J. Biol. Chem., 1983: 265-275.

Mahida,D. and Sendhil, R. 2017. Principal Component Analysis (PCA) based
Indexing. e-compendium of Data Analysis Tools and Approaches (DATA) in Agricultural Sciences. ICAR-Indian Institute of Wheat and Barley Research. pp 54-56.

Mallikarjunappa, R. S., Venkateshaiah, H. V., Rao, M. S. E., Anantharaman, M. N. and Bongale, U. D. 2008. Genetic variability and correlation studies in mulberry germplasm. Indian J. Seric., 47(2):226229.

Massey, W. F., 1965. Principal components regression in exploratory statistical research. J. Amer. Statist. Assoc., 60, 234-246.

Mohammadi, SA. 2003. Analysis of genetic diversity in crop plants salient statistical tools and considerations. Crop sci., 3 (4): 1235 .

Morris, D.L. 1948, Quantitative Determination of Carbohydrates With Dreywood's Anthrone Reagent. Science,107: 254 255pp.

Muniraja, C., Satish, R.G., Raju, C. and Hart, M., 2011. Principal component analysis among genotypes of chickpea (Cicer arietinum L.). Intern. J. Agri. Sci., 7(2), 382-386.

Oliveira, E. J. and Godoy, I. J. 2006. Pod yield stability analysis of runner peanut lines using AMMI. Crop Breeding and Applied Biotech. 6: 311-317.

Parpiev, B.A., 1968. Water metabolism in silkworms fed with a different strain changing diet. Sheik, 39: 15-17.

Purchase, J. L. 1997.Parametric analysis to described $G \times$ E interaction and yield stability in winter yield. Ph.D. Thesis. Department of Agronomy, Falculty of Agriculture, University of Orange Free State, Bloemfontein, South Africa.4-83.

Purchase, J. L., Hatting, H. and Vandeventer, C. S. 2000.Genotype $\times$ environment interaction of winter wheat (Triticumaestivum L.) in South Africa. II. Stability analysis of yield performance. South African J. Plant Soil,17: 101-107.

Purohit, K. M. and PavanKumar, T. 1996. Influence of various agronomical 
practices in India on the leaf quality in mulberry. A review. Sericologia, 36(1):27-39.

R Development Core Team- 2015. R: A Language and Environment for Statistical Computing. R Foundation for Statistical Computing, Vienna, Austria. ISBN 3900051- 07-0, URL http://www.Rproject.org.

Rahman, M. S. and Islam, S. M. S. 2020. Genetic variability and correlation studies of mulberry (Morus alba L.) genotypes in Bangladesh. Bangladesh J. Bot., 49(3): 685-691,

Ramamoorthy, R., Krishnakumar, N. and Paramanantham, M. 2018. Comparative biochemical study of improved mulberry (Morus indica) cultivars. I. J. Chem. Studies., 6(4): 1211-1213.

Ray, D., Mondal, L.N., Pain, A. K. and Mondal, S. K. 1973.Effect of NPK on farmyard manure on the yield and nutritive value of mulberry leaf. Indian J. Seri., 12, 7-12.

Richards, R. A., Rebetzke, G. J., Condon, A. G. and Herwaarden, A. F. V. 2002. Breeding opportunities for increasing the efficiency of water use and crop yield in temperate cereals. Crop Sci., 42: 111-121.

Salcedo, E. M. S., Amorós, A., Hernández, F. and Martínez, J. J. 2017. Physicochemical properties of white (Morus alba) and black (Morus nigra) mulberry leaves, a new food supplement. J. Food Nutrition Res., 5 (4):253-261.

Sheoran, O. P. Statistical Package for Agricultural Scientists (OPSTAT)," CCS HAU.

Shivwanshi, R. and Babbar, A., 2017. Principal Component Analysis of Chickpea Cicerarietinum L.) Germpasm. Int. J.
Curr. Microbiol. App. Sci., 6(10): 166173.

Sujathamma, P. and Dandin, S. B. 2000. Leaf quality evaluation of mulberry (Morus spp.) genotypes through chemical analysis. Indian J. Seric., 39(2): 117-121.

Suresh, K., Jalaja, S. K., Banerjee, R. and Trivedy, K. 2017. Genetic variability, correlation and path analysis in physiological and yield attributes in mulberry (Morus spp.). J. Crop Weed, 13(1):28-33.

Thirumalaisamy, R., Gowrishankar, J., Suganthapriya, S., Prakash, B., Kumar, A. L., and Arunachalam, G. 2009. Genetic variability in Morus alba L. by biochemical and bioassay methods for increased silk productivity. J. Biomed. Sci. Res., 1, 11-18.

Tikader, A. and Roy, B. N., 2001. Multivariate analysis in some mulberry (Morus spp.) germplasm accessions. Indian J. Seric., 40(2):168-170.

Tikader, A., Thangavelu, K. and Rao, A. A. 2004. Characterization and evaluation of mulberry (Morus spp.) germplasm. Indian J. Seric., 43(1):106-110.

Vijayashekara, Y. B. 2009. Genetic variation and character association in different accessions of mulberry (Morus sp.). M.Sc. Thesis, University of Agricultural Sciences, Bangalore. pp. 1-161.

Vikram, A. and Roy, D. 2003. Selection of characters for constructing selection index in ground nut (A. hypogea L.). Legume Res., 26(2): 137-139.

Xie, C., Xu, S. and Mosjidis, J. A. 1998. Multistage selection indices for maximum genetic gain and economic efficiency in red clover. Euphytica, 98(1-2): 75-82.

\section{How to cite this article:}

Suresh, K., H. Yallappa, Anil Pappachan, Maloy Laskar, G. R. Manjunath, D. Chakravarthy and Sivaprasad, V. 2021. Selection of Mulberry Genotypes for Rainfed Conditions through Principal Component Analysis. Int.J.Curr.Microbiol.App.Sci. 10(01): 2762-2778. doi: https://doi.org/10.20546/ijcmas.2021.1001.320 